\title{
Beware of Early Relapse in Rectal Cancer Patients Treated With Preoperative Chemoradiotherapy
}

\author{
Seul Gi Oh${ }^{1}$, In Ja Park², Ji-hyun Seo ${ }^{3}$, Young Il Kim², Seok-Byung Lim², Chan Wook Kim², Yong Sik Yoon², \\ Jong Lyul Lee ${ }^{2}$, Chang Sik Yu², Jin Cheon Kim² \\ Departments of ${ }^{1}$ Surgery and ${ }^{2}$ Colon and Rectal Surgery, Asan Medical Center, University of Ulsan College of Medicine, Seoul; ${ }^{3}$ Division of \\ Colorectal Surgery, Department of Surgery, Korea University Guro Hospital, Korea University College of Medicine, Seoul, Korea
}

Purpose: Recurrence patterns in rectal cancer patients treated with preoperative chemoradiotherapy (PCRT) are needed to evaluate for establishing tailored surveillance protocol.

Methods: This study included 2,215 patients with locally-advanced mid and low rectal cancer treated with radical resection between January 2005 and December 2012. Recurrence was evaluated according to receipt of PCRT; PCRT group $(\mathrm{n}=1,258)$ and no-PCRT group $(\mathrm{n}=957)$. Early recurrence occurred within 1 year of surgery and late recurrence after 3 years. The median follow-up duration was $65.7 \pm 29$ months.

Results: The overall recurrence rate was similar between the PCRT and no-PCRT group (25.8\% vs. 24.9\%, P =0.622). The most common initial recurrence site was the lungs in both groups ( $50.6 \%$ vs. $49.6 \%, \mathrm{P}=0.864)$, followed by the liver, which was more common in the no-PCRT group $(22.5 \%$ vs. $33.6 \%, \mathrm{P}=0.004)$. Most of the recurrence occurred within 3 years after surgery in both groups $(85.3 \%$ vs. $85.8 \%, \mathrm{P}=0.862)$. Early recurrence was more common in the PCRT group than in the no-PCRT group $(43.1 \%$ vs. $32.4 \%, \mathrm{P}=0.020)$. Recurrence within the first 6 months after surgery was significantly higher in the PCRT group than in the no-PCRT group $(18.8 \%$ vs. $7.6 \%, \mathrm{P}=0.003)$. Lung $(\mathrm{n}=27,44.3 \%)$ and liver $(\mathrm{n}=22,36.1 \%)$ were the frequent the first relapsed site within 6 months after surgery in PCRT group.

Conclusion: Early recurrence within the first 1 year after surgery was more common in patients treated with PCRT. This difference would be considered for surveillance protocols and need to be evaluated in further studies.

Keywords: Rectal neoplasms; Early recurrence; Preoperative chemoradiotherapy; Surveillance; Recurrence pattern

\section{INTRODUCTION}

Preoperative chemoradiotherapy (PCRT) is widely accepted as one of the most effective treatments for rectal cancer. It is considered the standard treatment for locally-advanced rectal cancer along with total mesorectal excision [1-3]. Many studies docu-

Received: April 16, 2020 • Accepted: June 11, 2020

Correspondence to: In Ja Park, M.D.

Department of Colon and Rectal Surgery, Asan Medical Center, University of Ulsan College of Medicine, 88 Olympic-ro 43-gil, Songpa-gu, Seoul 05505, Korea

Tel: +82-2-3010-3937, Fax: +82-2-3010-6701

E-mail: ipark@amc.seoul.kr

ORCID: https://orcid.org/0000-0001-5355-3969

(C) 2020 The Korean Society of Coloproctology

This is an open-access article distributed under the terms of the Creative Commons Attribution NonCommercial License (https://creativecommons.org/licenses/by-nc/4.0) which permits unrestricted noncommercial use, distribution, and reproduction in any medium, provided the original work is properly cited. mented significant improvement in local control and downstage of primary tumor in patients treated with PCRT [4-6]. It has been extended for practical use in locally-advanced rectal cancer and even lower clinical stage. Distant relapse, however, is still common despite improved and tailored treatment modalities $[3,7,8]$. Detecting recurrences at proper timing when curative treatment was possible effectively is important to improve oncologic outcomes even under neoadjuvant treatment era. Therefore, proper surveillance program in terms of surveillance method and timing based on recurrence pattern is critical.

There has been suspicion that recurrence patterns would be different in patients who received neoadjuvant treatment because it might alter tumor biology or discriminate "favorable" tumors according to tumor response. Several studies have evaluated recurrence pattern in rectal cancer treated with PCRT treatment [9-13]. The local recurrence occurred later in patients in the PCRT group than in patients who did not receive PCRT in some studies [11- 
13]. However, distant metastasis rate was not different according to PCRT although lung metastasis was more common in the PCRT group $[11,14]$. Frequent lung metastasis would be caused by more inclusion of low rectal cancer patients in the PCRT group.

Because the recurrence pattern after PCRT was not elucidated yet, a postoperative surveillance protocol has been used for patients with rectal cancer regardless of PCRT treatment. Current postoperative surveillance program was based on observations of rectal cancer patients not receiving PCRT. Therefore, we need to evaluate recurrence in patients who received PCRT thoroughly to apply proper surveillance protocol to these patients.

The aim of this study was to investigate recurrence in terms of sites and timing in locally-advanced rectal cancer patients treated with PCRT and showed difference from that of patients who did not receive PCRT.

\section{METHODS}

\section{Study population}

We retrospectively analyzed 2,215 patients with locally-advanced mid ( $>5 \mathrm{~cm}$ but $\leq 10 \mathrm{~cm}$ from the anal verge) or low $(\leq 5 \mathrm{~cm}$ from the anal verge) rectal cancer treated with radical resection between January 2005 and December 2012 at the Asan Medical Center, Seoul, Korea. Patients were categorized into the PCRT or no-PCRT group according to whether they received PCRT before surgery. Patients with concurrent distant metastasis at diagnosis, those with concurrent or prior malignancies within 5 years of the diagnosis of rectal cancer, or with prior history of immunotherapy or radiotherapy to the pelvis were excluded. Patients with no identifiable exact clinical stage and pathologic stage were also excluded (Fig. 1). This study was approved by the Institutional Review Board of Asan Medical Center and waived informed consent (2017-1114).

\section{Clinical diagnosis and treatment}

Clinical stage II or III diseases which were diagnosed as cT3/4 or node positive using computed tomography $(\mathrm{CT})$, magnetic resonance imaging (MRI), or transrectal ultrasonography was defined as locally-advanced rectal cancer.

Patients with suspicious mesorectal fascia involvement were recommended PCRT primarily. Patients who had obstructive lesion were recommended upfront surgical resection. Patients were given thorough information regarding the disease status, possible advantage/side effect of PCRT, and expected response rate and involved in decision of treatment plan. For patients who were decided to receive PCRT, a dose of 45 to $50.4 \mathrm{~Gy}$ of radiation therapy was given in 25 to 28 fractions to a target volume including the primary tumor, the perirectal adipose tissue, the lateral pelvis, and the presacral lymph node during the PCRT treatment period. As a part of a clinical trial, some patients received titanium silicate-1 concurrently with chemotherapy. Surgical resection was per-

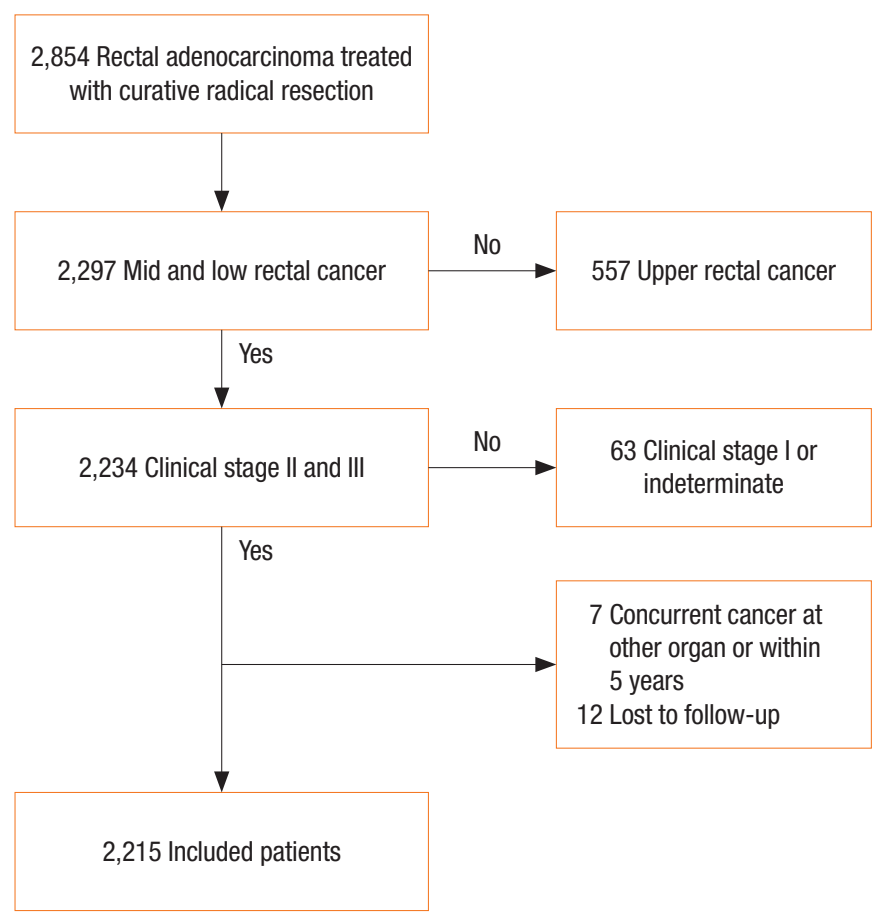

Fig. 1. Flow chart that describes inclusion of patients.

formed 6 to 8 weeks after completion of the radiation therapy. Adjuvant chemotherapy is recommended for all medically fit patients treated with PCRT. The standard adjuvant regimen consisted of 4 cycles of 5-fluorouracil and leucovorin (FL) monthly or 6 cycles of capecitabine. Patients were also treated with oxaliplatin at the discretion of the attending physician.

For patients who did not receive PCRT, radical surgical resection was performed according to the principle of total mesorectal excision. Postoperative chemoradiotherapy (CRT) was recommended to pathologic stage III patients. Postoperative CRT consisted of a total of 45 to 50.4 Gy of radiotherapy combined with FL. For patients with pathologic stage II disease, postoperative chemotherapy was recommended.

\section{Surveillance and oncologic outcomes}

All patients underwent follow-up examination every 3 to 6 months after surgery. This included patient history, physical examination, measurement of serum carcinoembryonic antigen levels. Abdominal, pelvic, and chest CT scans were performed every 6 months. Colonoscopy was performed every 2 to 3 years. When patients had obstructive lesion, colonoscopy was done within 6 months after surgical resection. Recurrences were diagnosed based on imaging modality and confirmed pathologically by biopsy if possible. When pathologic confirmation was not possible, diagnosis of recurrence was made combining more than 2 imaging modality diagnosis or serial change on same imaging method. Local recurrence was defined as the presence of a suspi- 
cious lesion in the pelvis (the site of anastomosis, the bed of the primary resection, etc.) identified by colonoscopy or imaging modalities such as abdominopelvic CT, MRI, or positron emission tomography (PET). Distant metastasis was defined as the presence of recurrence beyond the pelvis. The timing of recurrence was determined as the interval between the date of resection of the primary tumor and the date of diagnosis of recurrence. Early recurrence was defined as recurrence that developed within the first 1 year of surgery. Recurrence occurring later than 3 years after surgery was defined as late recurrence.

\section{Statistical analysis}

Categorical variables were compared using the chi-squared tests, and Student $\mathrm{t}$-tests were used for analysis of normally distributed continuous data. Relationships between independent variables and the time to event were established using the Kaplan-Meier method and compared by log-rank testing. Logistic regression analysis was performed for univariate and multivariate analyses of factors associated with early recurrence in the whole cohort and PCRT group separately. The covariates used in the analysis were PCRT, age, sex, clinical stage, location of tumor, pathologic T category, pathologic node metastasis, lymphovascular invasion (LVI), perineural invasion (PNI), pathologic circumferential resection margin (CRM) involvement, and sphincter-saving resection. Variables with $\mathrm{P}<0.2$ were used in the multivariate analysis. Statistical significance was assumed at the $5 \%$ level. All analyses were performed with IBM SPSS Statistics for Windows, ver. 21.0 (IBM Corp., Armonk, NY, USA).

\section{RESULTS}

\section{Clinicopathological characteristics of patients}

A total of 2,215 patients who were treated for locally-advanced rectal cancer during the study period were included. The median follow-up time was $65.7 \pm 29$ months. The no-PCRT group was older than the PCRT group. PCRT group had more patients with low rectal cancer $(\mathrm{P}<0.001)$ and clinical stage III disease $(\mathrm{P}<$ $0.001)$. Sphincter preservation was more frequently possible in the no-PCRT group than in the PCRT group $(\mathrm{P}<0.001)$. The proportion of the tumor contained within the bowel wall ([y] pT0-2) was higher in the PCRT group $(\mathrm{P}<0.001)$. Both LVI and PNI were more prevalent in the PCRT group (Table 1).

\section{Recurrence rate and initial recurrence site according to PCRT} The overall recurrence rate was similar between 2 groups (Table 2) and the 5-year disease-free survival rate was also not different between PCRT $(73.7 \%)$ and no-PCRT $(73.3 \%)$ group $(\mathrm{P}=0.685)$ (Fig. 2). Systemic relapse was the most common type of recurrence. In both groups, the lung was the most frequent site of initial recurrence ( $50.6 \%$ vs. $49.6 \%, \mathrm{P}=0.864$ ), followed by the liver (Fig. 3A). The proportion of liver metastases among initial recurrences was significantly lower in the PCRT group than in the no-
Table 1. Clinicopathological characteristics of the patients

\begin{tabular}{|c|c|c|c|}
\hline Variable & $\begin{array}{c}\text { PCRT } \\
(n=1,258)\end{array}$ & $\begin{array}{l}\text { No-PCRT } \\
(\mathrm{n}=957)\end{array}$ & P-value \\
\hline Age (yr) & $58 \pm 10.6(25-83)$ & $60.6 \pm 11.4(21-87)$ & $<0.001$ \\
\hline Sex & & & 0.005 \\
\hline Male & $845(67.2)$ & $587(61.3)$ & \\
\hline Female & $413(32.8)$ & $370(38.7)$ & \\
\hline Location of tumor & & & $<0.001$ \\
\hline Midrectum & $451(35.9)$ & 748 (78.2) & \\
\hline Lower rectum & $807(64.1)$ & $209(21.8)$ & \\
\hline Clinical stage & & & $<0.001$ \\
\hline Stage II & $68(5.4)$ & $136(14.2)$ & \\
\hline Stage III & $1,190(94.6)$ & $821(85.8)$ & \\
\hline Sphincter preservation & $965(76.7)$ & $883(92.3)$ & $<0.001$ \\
\hline Examined LNs & $16.1 \pm 7.4(0-64)$ & $21.3 \pm 9.6(0-58)$ & $<0.001$ \\
\hline pT category & & & $<0.001$ \\
\hline урT0-2 & $608(48.3)$ & $82(8.6)$ & \\
\hline урТ3-4 & $647(51.4)$ & $872(91.1)$ & \\
\hline pN category & & & $<0.001$ \\
\hline ypNO & $898(71.4)$ & $403(42.1)$ & \\
\hline ypN1 & $266(21.1)$ & $338(35.3)$ & \\
\hline ypN2 & $94(7.5)$ & $216(22.6)$ & \\
\hline Lymphovascular invasion & $612(48.6)$ & $365(38.1)$ & $<0.001$ \\
\hline Perineural invasion & $609(48.4)$ & $340(35.5)$ & $<0.001$ \\
\hline Follow-up duration (mo) & $66.5 \pm 27.9(5-156)$ & $66 \pm 31.6(6-159)$ & 0.690 \\
\hline Adjuvant chemotherapy & $1,143(90.9)$ & $677(70.7)$ & $<0.001$ \\
\hline
\end{tabular}

Values are presented as mean \pm standard deviation (range) or number (\%). PCRT, preoperative chemoradiotherapy; LN, lymph nodes.

Table 2. Recurrence pattern according to treatment with preoperative chemoradiotherapy

\begin{tabular}{lccc}
\hline Variable & $\begin{array}{c}\text { PCRT } \\
(\mathrm{n}=1,258)\end{array}$ & $\begin{array}{c}\text { No-PCRT } \\
(\mathrm{n}=957)\end{array}$ & P-value \\
\hline Recurrence & $325(25.8)$ & $238(24.9)$ & 0.622 \\
Time to recurrence (mo) & $20.3 \pm 17.3$ & $21.3 \pm 14.9$ & 0.472 \\
Recurrence type & & & 0.165 \\
$\quad$ Local recurrence & $47(14.5)$ & $22(9.2)$ & \\
Systemic metastasis & $264(81.5)$ & $207(87.0)$ & \\
Local + systemic & $13(4.0)$ & $9(3.8)$ & \\
\hline
\end{tabular}

Values are presented as mean \pm standard deviation (range) or number (\%). PCRT, preoperative chemoradiotherapy.

aPercentage among recurrences.

PCRT group (22.5\% vs. 33.6\%, $\mathrm{P}=0.004)$. Peritoneal seeding was observed more frequently as the initial recurrence site in the noPCRT group ( $2.8 \%$ vs. $5.9 \%, \mathrm{P}=0.084)$, but it was not statistically 


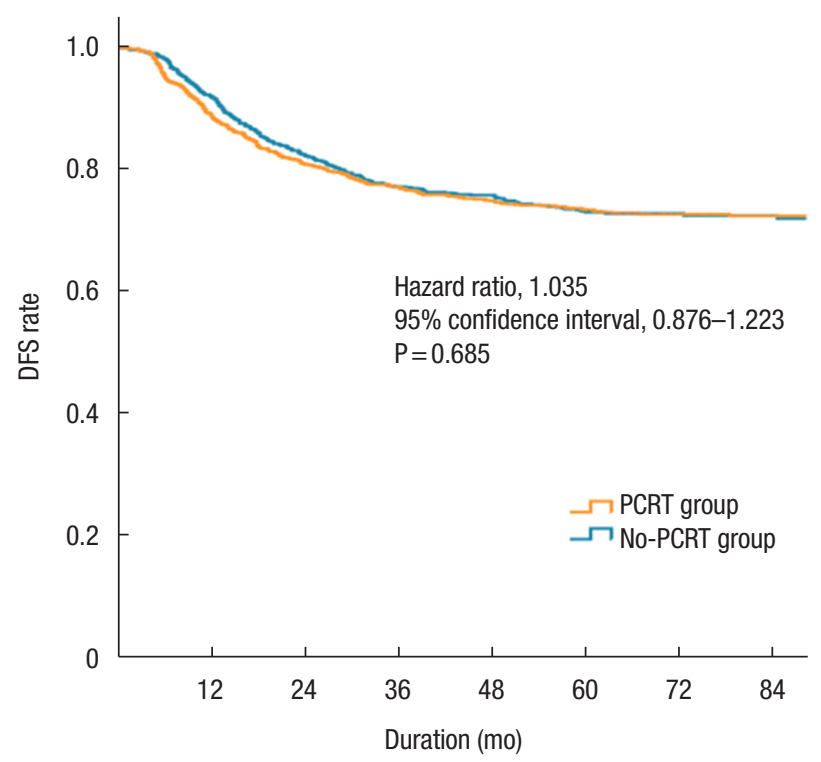

Fig. 2. The 5-year disease-free survival (DFS) rate according to treatment with preoperative chemoradiotherapy (PCRT).

significant. Local recurrence occurred more commonly as the initial recurrence site in the PCRT group compared with the noPCRT group ( $18.5 \%$ vs. $12.2 \%, \mathrm{P}=0.047)$.

Early recurrence sites were different in the 2 groups. The lungs (45.0\%) were the most common site of early recurrence, followed by the liver (27.9\%) in the PCRT group. However, both the liver (49.4\%) and lungs (45.5\%) were the most common sites of early recurrence in the no-PCRT group. Metastases at distant lymph nodes were significantly more common as early metastases in the PCRT group than in the no-PCRT group ( $16.4 \%$ vs. $6.4 \%$, $\mathrm{P}=0.055$; Fig. 3B).

There were no significant differences between the groups in late recurrence sites. The lungs were the most common site of late recurrence in the PCRT group. The rates of liver metastases (20.8\%) and local recurrence $(20.8 \%$ ) were similar in the PCRT group (Fig. 3C). After 5 years, only 2 local recurrences were detected, both in the PCRT group (2.5\%). The frequency of early and late recurrence sites in both groups was similar.

\section{Recurrence timing according to PCRT treatment}

Mean time to recurrence was not different between groups (Table 2). Mean time to local and distant relapse were also not different ( 20 vs. 21 months; $\mathrm{P}=0.922$ ). Patients were categorized based on the time to recurrence ( $\leq 6$ months, $>6$ months and $\leq 1$ year, $>1$ year and $\leq 2$ years, $>2$ years and $\leq 3$ years, $>3$ years and $\leq 5$ years, and $>5$ years) (Fig. 4). The rate of recurrence within 6 months was significantly higher in the PCRT group than in the no-PCRT group $(18.8 \%$ vs. $7.6 \%, \mathrm{P}<0.001)$. After 5 years, recurrence in the PCRT group was higher than that in the no-PCRT group, but this difference was not statistically significant $(P=0.187)$. Early recur-
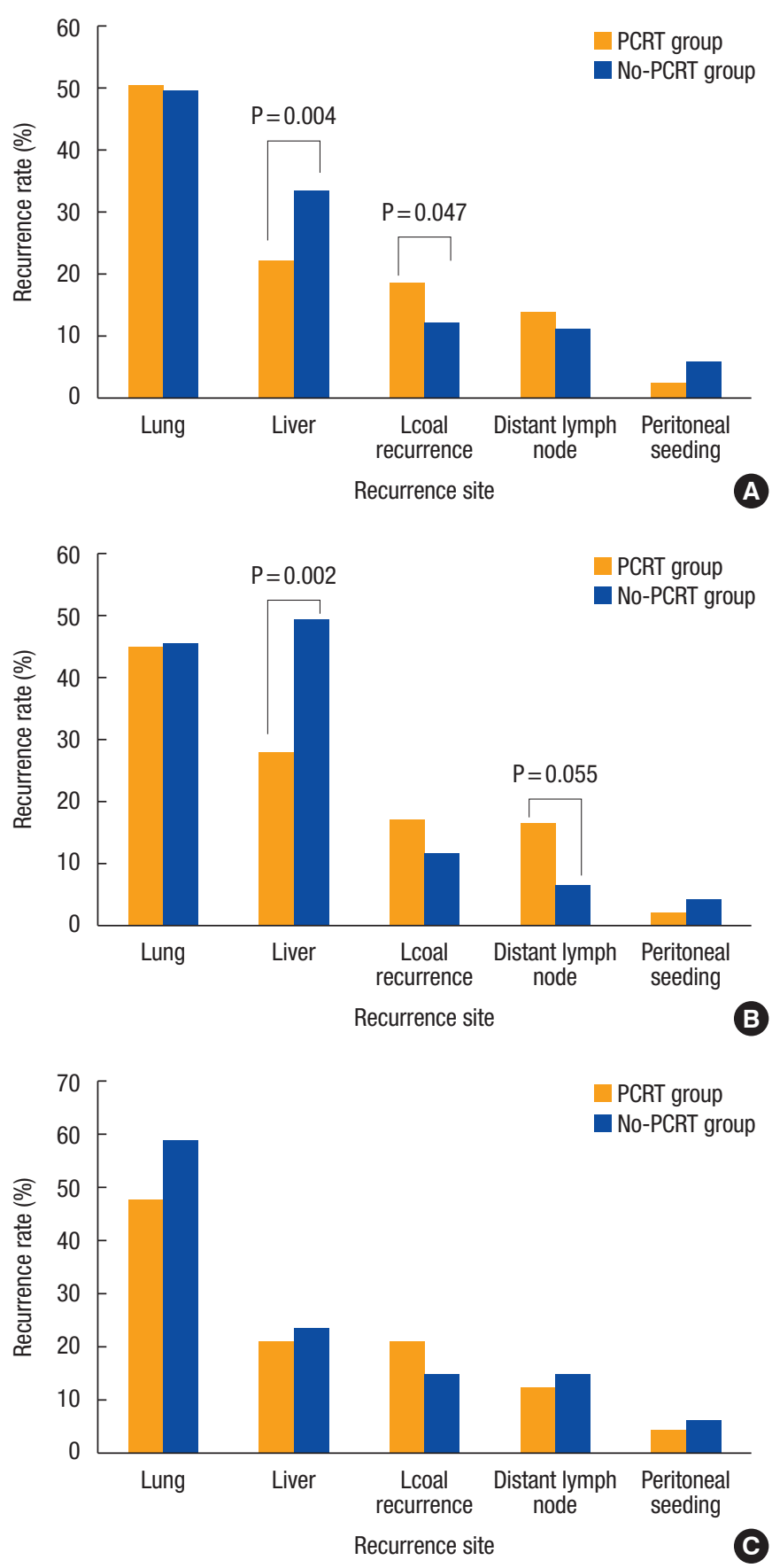

Fig. 3. The first recurrence site according to receipt of preoperative chemoradiotherpay (PCRT). (A) Overall. The no-PCRT group showed a higher rate of liver metastases among all types of recurrence than the PCRT group. (B) Early recurrence within the first year of operation. Among all recurrence sites, the rate of distant lymph node metastases was higher in the PCRT group than in the no-PCRT group. In the no-PCRT group, the liver was the common recurrence site for early recurrence. (C) Late recurrence after 3 years of operation. Distribution of the recurrence sites did not show a significant difference between the PCRT and no-PCRT groups. 
rence (within the first 1 year after surgery) was significantly higher in the PCRT group than in the no-PCRT group (43.1\% vs. $32.4 \%, \mathrm{P}=0.020$ ).

Recurrence patterns were evaluated by tumor location. In patients with mid-rectal cancer, the overall recurrence rate was similar between the groups (PCRT 24.8\%, no-PCRT 24.5\%, P = 0.890), but the proportion of early recurrence was significantly higher in the PCRT group than in the no-PCRT group (46.4\% vs. 33.3\%, $\mathrm{P}=0.027$ ). The proportion of early recurrence was not significantly different for patients with low rectal cancer (PCRT 41.3\%

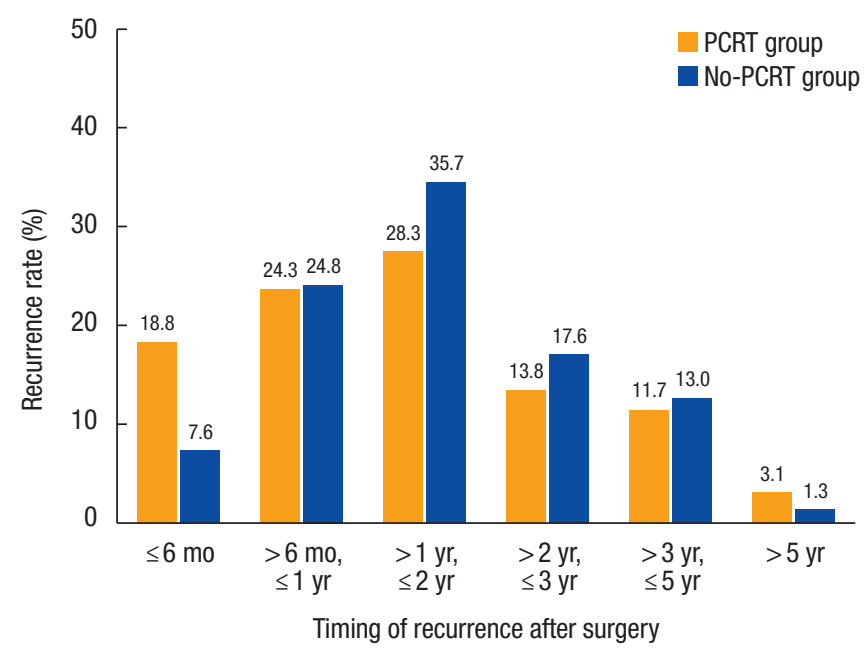

Fig. 4. Timing of recurrence according to treatment with preoperative chemoradiotherapy (PCRT). vs. no-PCRT $29.1 \%, \mathrm{P}=0.121)$, and the overall recurrence rate was similar between the groups (PCRT $26.4 \%$ vs. no-PCRT $26.3 \%, \mathrm{P}<0.999$ ).

Frequent early recurrence in the PCRT group was mainly due to early distant metastases. Among recurrences, the early recurrence rate for local recurrence did not show a difference between the PCRT (40.0\%) and no-PCRT (30.9\%) groups $(\mathrm{P}=0.486)$. However, the proportion of early recurrence rate for distant metastasis among overall recurrences was significantly higher in the PCRT group (43.7\%) than in the no-PCRT group (32.9\%) $(\mathrm{P}=0.015)$.

\section{Factors associated with early recurrence}

It is showed that patients who received PCRT were significantly related to early recurrence. Sphincter preservation showed negative association with early recurrence, and pathologic node metastasis was a factor of early recurrence in overall patients (Table 3). In the PCRT group, risk factors of early recurrence were analyzed separately. Pathologic node metastasis was significantly associated with early recurrence. Sphincter preservation showed also negative association with early recurrence in the PCRT group (Table 3). Location of tumor, clinical stage, sex, pathologic T category, LVI, PNI, and CRM involvement were determined as factors associated with early recurrence in the PCRT group as well as overall patients.

\section{DISCUSSION}

In the present study, recurrence within the first year after surgical resection occurred significantly more often in patients treated

Table 3. Risk factors associated with early recurrence, multivariate analysis

\begin{tabular}{|c|c|c|c|c|c|c|}
\hline \multirow{2}{*}{ Variable } & \multicolumn{3}{|c|}{ Overall patients } & \multicolumn{3}{|c|}{ PCRT group } \\
\hline & $\mathrm{HR}$ & $95 \% \mathrm{Cl}$ & P-value & $\mathrm{HR}$ & $95 \% \mathrm{Cl}$ & P-value \\
\hline PCRT & & & 0.017 & & & \\
\hline No & 1 & & & & & \\
\hline Yes & 1.593 & $1.085-2.340$ & & & & \\
\hline Sex & & & & & & 0.112 \\
\hline Male & & & & 1 & & \\
\hline Female & & & & 0.149 & $0.911-2.437$ & \\
\hline ypT stage & & & 0.309 & & & \\
\hline урт0-2 & 1 & & & & & \\
\hline урТ3-4 & 0.776 & $0.475-1.266$ & & & & \\
\hline ypN stage & & & 0.007 & & & 0.031 \\
\hline ypNO & 1 & & & 1 & & \\
\hline ypN+ & 1.696 & $1.158-2.484$ & & 1.655 & $1.046-2.618$ & \\
\hline SSR & 0.584 & $0.383-0.890$ & 0.003 & 0.469 & $0.286-0.768$ & 0.003 \\
\hline CRM involvement & 1.545 & $0.816-2.924$ & 0.182 & & & \\
\hline
\end{tabular}

HR, hazard ratio; Cl, confidence interval; PCRT, preoperative chemoradiotherapy; SSR, sphincter-saving resection; CRM, circumferential resection margin. 
with PCRT than in those not treated with PCRT and lung was predominant recurrence site. Although overall recurrence rate was similar between 2 groups, recurrence site and timing showed difference.

The recurrence pattern in patients treated with PCRT was suspected of being different from that of patients who did not receive PCRT. PCRT has been shown to effectively suppress local micrometastasis by improving tumor oxygenation $[4,15]$. Therefore, tumor regrowth and recurrence would be expected to develop later in patients treated with PCRT. Some studies reported that local recurrence developed later in the PCRT group than in the no-PCRT group. Yeo et al. [11] reported that the rate of overall recurrence was $23.1 \%$ in patients who received PCRT and $30.7 \%$ in patients who received postoperative CRT. The time to local failure in the PCRT group was longer than in the postoperative CRT group $(37.2 \pm 21.2$ vs. $35.2 \pm 27.4$ months, $\mathrm{P}=0.841)$ although it was not significant. Marin et al. [12] reported that local recurrence occurred significantly later in patients who underwent PCRT than in those who did not receive PCRT (51.8 vs. 13.5 months, $\mathrm{P}=0.002$ ). Coco et al. [16] reported $28 \%$ of all local recurrence after the first 5 years after surgery in patients treated with PCRT.

The present study report that time to local recurrence was not different between the PCRT and no-PCRT groups. Although the local recurrence rate was higher in patients who received PCRT than in those who did not receive PCRT, the proportion of early and late local recurrence was similar. We defined late recurrence as recurrence occurred after 3 years from surgery because $85.4 \%$ of recurrence developed within the first 3 years after surgery. Within recommended surveillance period, local recurrence did not occur later in patients who received PCRT in the present study. Regular postoperative surveillance was conducted for up to 5 years after surgery, according to clinical treatment guidelines for colorectal cancer $[17,18]$. However, regular surveillance after 5 years was quite various according to disease status, economic status, performance status, and health care accessibility. Among living patients, $67.7 \%$ of patients were followed up longer than 5 years in the present study. It is less than the other studies which reported a longer time to local recurrence in patients treated with PCRT $[11,12]$. This would be influence on detection of local recurrence beyond postoperative 5 years.

The low rate of local recurrence may also affect results. The local recurrence rate in both groups was lower than $5 \%$ which was quite low rate considering reported local recurrence rate in the previous reports $[4,5,11,12]$. Furthermore, the rate of local recurrence after 5 years was $2.5 \%$ in the PCRT group, and there were no patients who relapsed beyond 5 years in the no-PCRT group. The limited number of patients in these categories may have skewed the statistical results. The local recurrence rate was higher in the PCRT group than in the no-PCRT group, although the difference was not statistically significant. This is contrary to results of current reports, which indicate that PCRT is more effective for local control. Although we included clinical stage II and III disease in both groups, compared to the no-PCRT group, the PCRT group had more aggressive features, such as threatened CRM on imaging modalities, bulky tumors, or cT4 diseases, which were not determined by the clinical stage $[19,20]$. Therefore, these features would influence the local relapse in the PCRT group.

In the present study, early recurrence in the PCRT group was mainly due to higher rates of early distant metastases than those in the no-PCRT group. Early distant recurrence after PCRT could be confused with interval failure, which occurs after starting PCRT, or metastases that existed but were not detected at the time of initial diagnosis. In this study, abdominal, pelvic, and chest CT were performed to detect distant metastasis, and PET-CT was also performed in the case of indeterminate lesions found on CT, in order to exclude preexisting distant metastasis before PCRT and reevaluated before surgical resection in PCRT group. Thus, we consider early recurrences in the PCRT group to represent newly developed lesions, but it is difficult to completely exclude the possibility of micrometastases precluded from detection with imaging.

Patients with advanced features related to poor prognosis [1820] might have been included in the PCRT group more frequently. LVI and PNI were more prevalent in the PCRT group than in the no-PCRT group in the present study. These adverse features are risk factors for early recurrence in patients treated with PCRT. Recently, there is increasing interest in the tailored application of PCRT according to detailed tumor characteristics. Aggressive features, such as a threatened CRM, the presence of an extramural venous invasion, and a more advanced T3 substage, indicate the need for PCRT [18]. These indications were used in the present study. More patients with advanced rectal cancer who are more prone to early recurrence would be more likely to receive PCRT according to recent tailored treatment strategies. Therefore, the present study result, which indicated that early recurrence within the first 6 months was significantly more likely in the PCRT group than in the no-PCRT group, is helpful for real clinical practice.

However, early relapse made us reconsider the effectiveness of adjuvant chemotherapy in a PCRT setting. The effects of adjuvant chemotherapy on oncologic outcomes in PCRT settings have not been defined thus far. Although the folinic acid, fluorouracil, and oxaliplatin (FOLFOX) adjuvant chemotherapy has been reported to improve disease-free survival in stage II/III rectal cancer after 5 fluoropyrimidine-based PCRT and radical resection in a study $[21,22]$, other randomized European trials have failed to show benefits of adjuvant chemotherapy on survival outcomes [23-26]. In addition, insufficient compliance rate was also one of limitation for demonstrating advantage of adjuvant chemotherapy in these trials. Therefore, total neoadjuvant therapy has been suggested to improve compliance with treatment. It also has the potential advantage of micrometastases control and improving pathological response [27]. Considering the higher early recurrence rate in patients treated with PCRT, total neoadjuvant therapy should be carefully considered for these patients and there is a 
need to discover the benefits/disadvantages and effects of further treatment on oncological outcomes.

In the present study, the lung was the most common initial recurrence site regardless of PCRT treatment. Liver metastasis, however, was significantly more common in the no-PCRT group. The primary tumor location is related to the distant metastasis site, and lung metastasis is reported to be more common in patients with rectal cancer, especially low rectal cancer [8-10, 28-30]. This was known due to differences in systemic circulation between the upper and the lower rectum $[14,29]$. Lower rectal cancer was more common in the PCRT than in the no-PCRT group, which could explain the relatively high rate of lung metastasis in the PCRT group. The patterns of recurrence at other sites also differed according to PCRT treatment. Distant lymph node recurrence was higher in the PCRT group, while, peritoneal seeding was detected more often among patients who did not receive PCRT. These findings could be used to improve the reliability of the surveillance program. Further studies are required to determine the utility of tailored surveillance according to PCRT treatment.

A limitation of the current study was the heterogeneity of the patients in each group. The PCRT group included more advanced cases than the no-PCRT group. Although we specifically included patients with locally-advanced rectal cancer with clinical stage II/ III, there might still be differences that could not be standardized using simple clinical staging. The main limitation of this retrospective cohort study was the possibility of biased information. Only $67.7 \%$ of living patients continued long-term surveillance after 5 years, which might not be of sufficient duration to reach conclusions regarding late recurrence. However, the proportions of patients receiving late surveillance were not different between the PCRT and the no-PCRT groups, making it possible to compare the late recurrence rate between the 2 groups.

Despite these limitations, we were able to evaluate the recurrence pattern according to timing and sites after surgical resection, and to describe the recurrence pattern of rectal cancer in real-world practice. In addition, we identified recurrence pattern of patients received PCRT would be different. This information may help clinicians to develop tailored surveillance protocols for rectal cancer patients who treated with PCRT, based on their specific recurrence patterns.

In conclusion, this study demonstrates the need for more careful detection of early recurrence in rectal cancer patients treated with PCRT. Intensive surveillance during the early period in these patients may lead to improved detection of recurrence. Additional comprehensive information regarding the pattern of recurrence in patients receiving PCRT will be required to recommend tailored surveillance protocol in these patients.

\section{CONFLICT OF INTEREST}

No potential conflict of interest relevant to this article was reported.

\section{ACKNOWLEDGMENTS}

This study was supported by a grant 'Elimination of Cancer Project Fund' from Asan Cancer Institute of Asan Medical Center, Seoul, Korea.

\section{REFERENCES}

1. Maurer CA, Renzulli P, Kull C, Käser SA, Mazzucchelli L, Ulrich $A$, et al. The impact of the introduction of total mesorectal excision on local recurrence rate and survival in rectal cancer: longterm results. Ann Surg Oncol 2011;18:1899-906.

2. Heald RJ, Ryall RD. Recurrence and survival after total mesorectal excision for rectal cancer. Lancet 1986;1:1479-82.

3. Bosset JF, Collette L, Calais G, Mineur L, Maingon P, RadosevicJelic L, et al. Chemotherapy with preoperative radiotherapy in rectal cancer. N Engl J Med 2006;355:1114-23.

4. Sauer R, Becker H, Hohenberger W, Rödel C, Wittekind C, Fietkau R, et al. Preoperative versus postoperative chemoradiotherapy for rectal cancer. N Engl J Med 2004;351:1731-40.

5. Sauer R, Liersch T, Merkel S, Fietkau R, Hohenberger W, Hess C, et al. Preoperative versus postoperative chemoradiotherapy for locally advanced rectal cancer: results of the German CAO/ARO/ AIO-94 randomized phase III trial after a median follow-up of 11 years. J Clin Oncol 2012;30:1926-33.

6. Roh MS, Colangelo LH, O'Connell MJ, Yothers G, Deutsch M, Allegra CJ, et al. Preoperative multimodality therapy improves disease-free survival in patients with carcinoma of the rectum: NSABP R-03. J Clin Oncol 2009;27:5124-30.

7. Tjandra JJ, Chan MK. Follow-up after curative resection of colorectal cancer: a meta-analysis. Dis Colon Rectum 2007;50: 1783-99.

8. Lee JL, Yu CS, Kim CW, Yoon YS, Lim SB, Kim JC. Chronological improvement in survival following rectal cancer surgery: a largescale, single-center study. World J Surg 2013;37:2693-9.

9. Ikoma N, You YN, Bednarski BK, Rodriguez-Bigas MA, Eng C, Das $P$, et al. Impact of recurrence and salvage surgery on survival after multidisciplinary treatment of rectal cancer. J Clin Oncol 2017;35:2631-8.

10. Frambach P, Pucciarelli S, Perin A, Zuin M, Toppan P, Maretto I, et al. Metastatic pattern and new primary tumours after neoadjuvant therapy and surgery in rectal cancer. Colorectal Dis 2018;20: O326-34.

11. Yeo SG, Kim MJ, Kim DY, Chang HJ, Kim MJ, Baek JY, et al. Patterns of failure in patients with locally advanced rectal cancer receiving pre-operative or post-operative chemoradiotherapy. Radiat Oncol 2013;8:114.

12. Marin G, Suárez J, Vera R, Balén E, Viudez A, Mata E. Local recurrence after five years is associated with preoperative chemoradiotherapy treatment in patients diagnosed with stage II and III rectal cancer. Int J Surg 2017;44:15-20.

13. Merkel S, Mansmann U, Hohenberger W, Hermanek P. Time to 
locoregional recurrence after curative resection of rectal carcinoma is prolonged after neoadjuvant treatment: a systematic review and meta-analysis. Colorectal Dis 2011;13:123-31.

14. Ding P, Liska D, Tang P, Shia J, Saltz L, Goodman K, et al. Pulmonary recurrence predominates after combined modality therapy for rectal cancer: an original retrospective study. Ann Surg 2012; 256:111-6.

15. Liefers GJ, Cleton-Jansen AM, van de Velde CJ, Hermans J, van Krieken JH, Cornelisse CJ, et al. Micrometastases and survival in stage II colorectal cancer. N Engl J Med 1998;339:223-8.

16. Coco C, Valentini V, Manno A, Mattana C, Verbo A, Cellini N, et al. Long-term results after neoadjuvant radiochemotherapy for locally advanced resectable extraperitoneal rectal cancer. Dis Colon Rectum 2006;49:311-8.

17. Benson AB, Venook AP, Al-Hawary MM, Cederquist L, Chen YJ, Ciombor KK, et al. Rectal cancer, version 2.2018, NCCN Clinical Practice Guidelines in Oncology. J Natl Compr Canc Netw 2018; 16:874-901.

18. Glynne-Jones R, Wyrwicz L, Tiret E, Brown G, Rödel C, Cervantes A, et al. Rectal cancer: ESMO Clinical Practice Guidelines for diagnosis, treatment and follow-up. Ann Oncol 2017;28(Suppl 4):iv22-40.

19. Hunter CJ, Garant A, Vuong T, Artho G, Lisbona R, Tekkis P, et al. Adverse features on rectal MRI identify a high-risk group that may benefit from more intensive preoperative staging and treatment. Ann Surg Oncol 2012;19:1199-205.

20. Taylor FG, Quirke P, Heald RJ, Moran BJ, Blomqvist L, Swift IR, et al. Preoperative magnetic resonance imaging assessment of circumferential resection margin predicts disease-free survival and local recurrence: 5-year follow-up results of the MERCURY study. J Clin Oncol 2014;32:34-43.

21. Hong YS, Nam BH, Kim KP, Kim JE, Park SJ, Park YS, et al. Oxaliplatin, fluorouracil, and leucovorin versus fluorouracil and leucovorin as adjuvant chemotherapy for locally advanced rectal cancer after preoperative chemoradiotherapy (ADORE): an openlabel, multicentre, phase 2, randomised controlled trial. Lancet Oncol 2014; 15:1245-53.

22. Hong YS, Kim SY, Lee JS, Nam BH, Kim KP, Kim JE, et al. Oxaliplatin-based adjuvant chemotherapy for rectal cancer after pre- operative chemoradiotherapy (ADORE): long-term results of a randomized controlled trial. J Clin Oncol 2019;37:3111-23.

23. Bosset JF, Calais G, Mineur L, Maingon P, Stojanovic-Rundic S, Bensadoun RJ, et al. Fluorouracil-based adjuvant chemotherapy after preoperative chemoradiotherapy in rectal cancer: long-term results of the EORTC 22921 randomised study. Lancet Oncol 2014;15:184-90.

24. Breugom AJ, van Gijn W, Muller EW, Berglund A, van den Broek $\mathrm{CB}$, Fokstuen T, et al. Adjuvant chemotherapy for rectal cancer patients treated with preoperative (chemo)radiotherapy and total mesorectal excision: a Dutch Colorectal Cancer Group (DCCG) randomized phase III trial. Ann Oncol 2015;26:696-701.

25. Glynne-Jones R, Counsell N, Quirke P, Mortensen N, Maraveyas A, Meadows HM, et al. Chronicle: results of a randomised phase III trial in locally advanced rectal cancer after neoadjuvant chemoradiation randomising postoperative adjuvant capecitabine plus oxaliplatin (XELOX) versus control. Ann Oncol 2014;25: 1356-62.

26. Sainato A, Cernusco Luna Nunzia V, Valentini V, De Paoli A, Maurizi ER, Lupattelli M, et al. No benefit of adjuvant fluorouracil leucovorin chemotherapy after neoadjuvant chemoradiotherapy in locally advanced cancer of the rectum (LARC): long term results of a randomized trial (I-CNR-RT). Radiother Oncol 2014; 113:223-9.

27. Sclafani F, Brown G, Cunningham D, Wotherspoon A, Tait D, Peckitt C, et al. PAN-EX: a pooled analysis of two trials of neoadjuvant chemotherapy followed by chemoradiotherapy in MRI-defined, locally advanced rectal cancer. Ann Oncol 2016;27:155765.

28. Lee JL, Yu CS, Kim TW, Kim JH, Kim JC. Rate of pulmonary metastasis varies with location of rectal cancer in the patients undergoing curative resection. World J Surg 2015;39:759-68.

29. Steup WH, Moriya Y, van de Velde CJ. Patterns of lymphatic spread in rectal cancer: a topographical analysis on lymph node metastases. Eur J Cancer 2002;38:911-8.

30. Popek S, Tsikitis VL, Hazard L, Cohen AM. Preoperative radiation therapy for upper rectal cancer T3,T4/Nx: selectivity essential. Clin Colorectal Cancer 2012;11:88-92. 